Review Article

\title{
Possible Retinal Impairment Secondary to Ritonavir Use in SARS-CoV-2 Patients: A Narrative Systematic Review
}

\author{
Grazia Maria Cozzupoli (D, , ${ }^{1,2}$ Maria Cristina Savastano, ${ }^{1,2}$ Benedetto Falsini,, ${ }^{1,2}$ \\ Alfonso Savastano, ${ }^{1,2}$ and Stanislao Rizzo ${ }^{1,2,3}$ \\ ${ }^{1}$ UOC Oculistica, Fondazione Policlinico Universitario A. Gemelli IRCCS, Rome 00168, Italy \\ ${ }^{2}$ Università Cattolica del Sacro Cuore, Rome 00168, Italy \\ ${ }^{3}$ Consiglio Nazionale delle Ricerche, Istituto di Neuroscienze, Pisa 56124, Italy
}

Correspondence should be addressed to Grazia Maria Cozzupoli; mgcozzupoli@gmail.com

Received 2 May 2020; Accepted 12 August 2020; Published 22 August 2020

Academic Editor: Tomasz Zarnowski

Copyright ( 2020 Grazia Maria Cozzupoli et al. This is an open access article distributed under the Creative Commons Attribution License, which permits unrestricted use, distribution, and reproduction in any medium, provided the original work is properly cited.

\begin{abstract}
Some reports described a possible ritonavir-related retinal toxicity. The objective of this research was to review and analyze previous studies conducted on ritonavir administration and retinal impairment in a narrative synthesis. PubMed was used to perform a systematic review of ritonavir effects and retinal damage. All studies up to December 2019 were considered. Seven single cases and one case series, reporting a total of 10 patients affected by retinal changes secondary to long-term ritonavir treatment, were included in the review. Variable degrees of outer retina and retinal pigment epithelium changes were detected in most of the patients, with two patients showing macular telangiectasia, four patients presenting intraretinal crystal deposits, two patients disclosing a bull's eye maculopathy, and two patients revealing midperipheral bone spicule-like pigment changes. In the present study, we hypothesized that the use of ritonavir in life-saving treatments of severe acute respiratory syndrome-coronavirus- 2 (SARS-CoV-2) pneumonia might expose these patients to the risk of developing a retinotoxicity. We aimed to alert ophthalmologists on the importance of recognizing ritonavir-induced retinal impairment in SARS-CoV-2 patients. These findings are the target for personalized medicine.
\end{abstract}

\section{Introduction}

Ritonavir is a human immunodeficiency virus type 1 (HIV1) protease inhibitor, approved by the Food and Drug Administration in 1996 to be used in the highly active antiretroviral therapy (HAART), which is recognized as the most effective treatment method for human immunodeficiency virus (HIV) patients.

In light of the benefits produced by ritonavir in combination with lopinavir in the treatment of severe acute respiratory syndrome and Middle East respiratory syndrome, the association lopinavir/ritonavir has been integrated in the treatment guidelines of 2019 novel coronavirus (2019-nCoV) infected pneumonia [1] with a weak recommendation level [2]. Although a recent randomized trial [3] involving hospitalized adult patients with confirmed severe acute respiratory syndrome-coronavirus-2 (SARS-CoV-2) infection has not shown significant benefits with lopinavir/ritonavir treatment beyond standard care, this protease inhibitor association is used in worldwide hospitals [4].

1.1. Mechanism of Action. As an HIV-1 protease inhibitor, ritonavir prevents the cleavage process of viral polyprotein precursors into mature and functional proteins, hence interrupting the production of new viral particles. Although ritonavir was initially designed to inhibit HIV-1 protease, studies have found that it also inhibits cytochrome P4503A4 (CYP450-3A4) and, to a lesser extent, cytochrome P450-2D6 (CYP450-2D6) [5, 6]. The CYP450-3A4 pathway metabolizes other protease inhibitors. For this reason, coadministration of low-dose ritonavir with other protease 
inhibitors enhances their bioavailability and allows lower dosing and less frequent administration of these drugs [5].

The most common side effects of ritonavir are nausea, vomiting, diarrhea, change in taste, fatigue, rash, and, with long-term use, hyperlipidemia and lipodystrophy. Retinal toxicity has been described by some authors in case reports and series, but it is a rare and little-known complication of ritonavir. We review the side effects of ritonavir in the human retina.

1.2. Ritonavir-Induced Retinal Toxicity. To the best of our knowledge, this is the first review of the retinal changes caused by ritonavir in HAART-treated patients. Although retinotoxicity secondary to ritonavir has not yet been investigated by pharmacokinetic and pharmacodynamic studies on humans, animal data submitted to the European Medicines Agency [7] have identified liver, retina, thyroid, and kidney as susceptible to dose-related ritonavir damage. Moreover, the same report described hypertrophy of the retinal pigment epithelium (RPE) and retinal degeneration in rodents treated with ritonavir and assumed that rodent liver and eye lesions due to ritonavir were related to phospholipidosis. In fact, the electron micrographs of both liver parenchyma and retina demonstrated the presence of amorphous granular inclusion bodies, characteristic of phospholipidosis, and this phenomenon appeared more predominant in the retina than in the liver.

In addition, a study [8] conducted on in vitro cellular models demonstrated that ritonavir can strongly inhibit hypoxia-inducible factor $1 \alpha$ and vascular endothelial growth factor (VEGF). Interestingly, VEGF knockout mice exhibit degeneration of the choriocapillaris-RPE complex and dysfunction of cone photoreceptors [9]. On the basis of this evidence, Kurihara et al. [9] postulated that VEGF might have a direct neurotrophic effect on photoreceptors in nonhypoxic retinas in addition to the well-known role in regulating endothelial cell function [10]. Therefore, VEGF inhibition by ritonavir should be considered as a further pathogenetic mechanism underlying the toxic damage to the choriocapillaris, RPE cells, and photoreceptors.

\section{Methods}

Because of the shortage of reports found in PubMed about the association between ritonavir and retinal toxicity, we adhered to the Preferred Items for Systematic Reviews and Meta-Analyses (PRISMA) guidelines [11], but we did not need to create a flow diagram.

2.1. Study Selection. A literature search was carried out on PubMed and The Cochrane Library. The search query included ritonavir AND (retina OR ocular OR retinal) AND (toxicity OR toxic OR changes OR impairment OR damage). The inclusion criteria for each study were as follows: [1] documented retinal impairment due to ritonavir treatment and [2] conducted in human eyes. All full-text selection was carried out by two authors (G.M.C. and M.C.S.) independently. Any disagreements were resolved by consensus with a third author (B.F).

2.2. Data Collection and Analysis. The small number of reference reports did not allow any statistical analyses for data correlation or comparison. However, the qualitative features of the retinal impairment were reported in detail in each included study. Thus, we conducted a narrative systematic review of the published reports describing the clinical features and imaging signs associated with ritonavirinduced retinal toxicity.

In order to establish the macular atrophy stage, we followed the new consensus definition for atrophy associated with age-related macular degeneration on OCT [12]. Images from each report were analyzed and graded by two readers (G.M.C. and M.C.S.) separately. In case of disagreement, a third author (B.F.) was consulted to reach consensus.

The quality of the reports was assessed using the Joanna Briggs Institute Critical Appraisal Checklist for case reports [13]. Two reviewers (G.M.C. and M.C.S.) rated the studies independently, and the final decision was reached by consensus with a third author (B.F.).

\section{Results and Discussion}

3.1. Results. The literature search yielded 9 potentially relevant publications. A total of 8 papers satisfied the predetermined inclusion criteria and were included in the review, 7 of which were single case reports and 1 was a case series including 3 patients. One case report was excluded since it did not provide exhaustive clinical details and imaging (Appendix A). The mean score of the Joanna Briggs Institute Critical Appraisal Checklist was 6. Overall, 10 patients affected by retinal abnormalities secondary to longterm ritonavir treatment were imaged and reported between 2011 and 2019 (Table 1). Ritonavir was administered at a dose of $100 \mathrm{mg}$ daily or twice daily $[14,15,20]$ in the context of a HAART regimen. The overall average treatment duration was 75 months. The mean age of the patients was 47 years and the patients were all male.

All reports dealing with chronic ritonavir-induced retinal toxicity were united by a common feature, which is the presence of macular pigmentary changes and outer retina impairment. In almost all described cases, ritonavir-related retinotoxicity presented at the fundus examination as a bilateral mottling or atrophy of the RPE affecting the macula, with unilateral macular involvement in one case [18] and involvement of either the macula and the peripheral retina in three cases $[17,20,21]$. Moreover, some peculiar clinical pictures were reported by different authors. Four cases were similar to the clinical picture of type 2 macular telangiectasia (Mac Tel type 2), two of which [14] presenting macular capillary telangiectasias and crystalline deposits, one other [14] revealing extensive macular atrophy, intraretinal cysts, and crystal deposits, and the fourth one [19] showing macular graying and both crystalline and pigment deposits, without evident telangiectasias. 


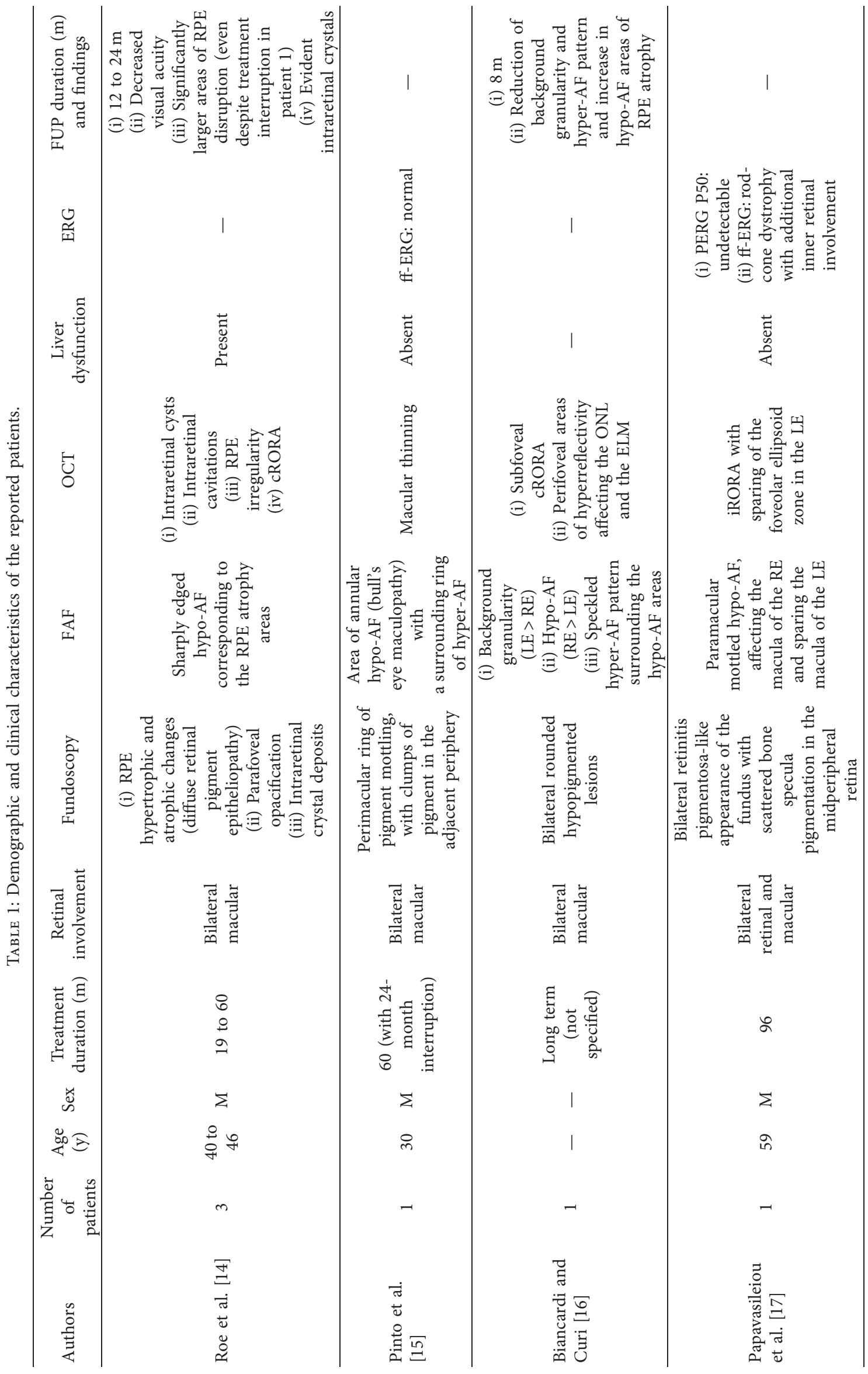




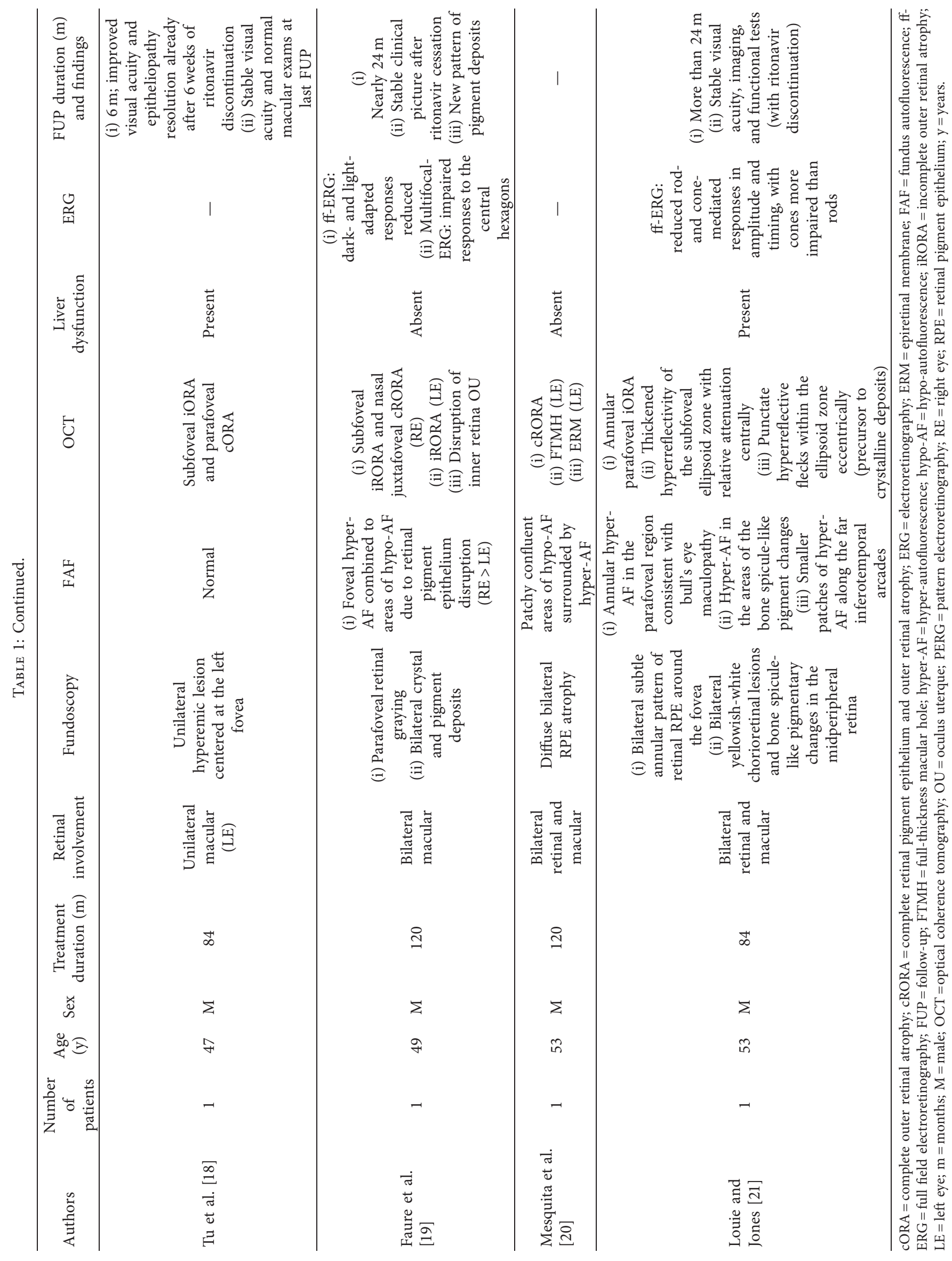


Two authors reported a bull's eye macular pattern $[15,21]$. In particular, Louie and Jones [21] described a patient with bull's eye maculopathy associated with bone spicule-like pigment changes in the midperipheral retina. This latter finding was observed as well in a case presenting as a pseudoretinitis pigmentosa [17].

No alterations of the optic disk and retinal vessels were found. The main optical coherence tomography (OCT) features shown by these patients were as follows:

(1) Complete RPE and outer retinal atrophy (cRORA) defined by a zone of homogeneous choroidal hypertransmission and absence of the RPE band with overlying outer retinal thinning and loss of photoreceptors $[16,19,20]$.

(2) Incomplete RPE and outer retinal atrophy (iRORA), with some discontinuous hypertransmission visible but irregular RPE band and photoreceptor degeneration $[17,19]$.

(3) Incomplete and complete outer retinal atrophy (iORA and CORA) characterized by visible and nonvisible external limiting membrane and ellipsoid zone, respectively, and paralleled with nondetectable interdigitation zone and severe thinning of the outer retina in the setting of an intact RPE band [18].

(4) Intraretinal cysts and cavitations [14].

(5) Epiretinal membrane (ERM) and full-thickness macular hole (FTMH) [20].

Corresponding to the areas of RPE atrophy, the fundus autofluorescence (FAF) showed a patchy hypo-autofluorescence (hypo-AF), surrounded by a speckled or annular hyper-autofluorescence (hyper-AF), attributed to the active toxicity of ritonavir $[16,20]$.

3.2. Discussion. As far as we are aware, this is the first systematic review of the literature concerning ritonavirinduced retinal changes.

There are many antiviral treatments currently used against SARS-CoV-2 infection for which ocular side effects (mainly related to conditions other that direct cellular toxicity, such as inflammatory responses, autoimmunity reactivation, and vascular damage) have been pointed out [22]. Unlike these antivirals, ritonavir appears to exert a toxic effect on the outer retina and RPE, causing their irreversible degeneration in the long term and threatening the central vision without therapeutic options available. Thus, we aimed to alert ophthalmologists on the possible retinal damage that might occur in some patients hospitalized for SARS-CoV-2 pneumonia and treated with ritonavir.

Indeed, we do not know yet if the administration time of life-saving therapies containing ritonavir in SARS-CoV-2infected patients is sufficiently long to produce a retinal impairment. The overall average treatment duration in the reported cases of HIV patients was 75 months in the context of a HAART regimen. Differently, in the Chinese trial cited above [3], SARS-CoV-2 patients received lopinavir-ritonavir
( $400 \mathrm{mg}$ and $100 \mathrm{mg}$, respectively) twice a day for 14 days. Given the brief duration of ritonavir treatment in SARSCoV-2 patients, it is unlikely that patients will develop a retinal toxicity, which is supposed to be dose- and timerelated [23]. Nonetheless, some polymorphisms, for example, on the genes encoding enzymes involved in drug hepatic clearance, and certain comorbidities could place specific subsets of patients at an increased risk for retinal changes. SARS-CoV-2 infection has been shown to involve mainly the respiratory system but also the liver and kidneys [24-26], impairing the metabolism and excretion of the medications used to treat the disease. On the one hand, liver dysfunction can reduce ritonavir hepatic clearance, increasing the total serum drug concentration and predisposing to more severe side effects. On the other hand, kidney injury can impair excretion, dosing, and expected concentrations of hydroxychloroquine, another drug currently used for the treatment of SARS-CoV-2 infection [27, 28] and given together with antiviral and supportive therapies. Hydroxychloroquine has a well-known dose- and time-related toxicity on RPE cells and photoreceptors [29, 30]. Furthermore, a case of short-term retinal toxicity induced by hydroxychloroquine has been recently described by Pasaoglu and Onmez [31]. Anyway, it is not illogical to hypothesize that the retinal toxic effects of both long-term hydroxychloroquine [32] and ritonavir therapies might appear also after short-term treatments, as in the case of SARS-CoV-2 patients, enhanced by the deterioration of renal and hepatic clearance function, respectively. Noteworthily, we cannot exclude that even subtle toxic changes caused by short-term hydroxychloroquine and ritonavir, independently, may add up because of their coadministration, giving a clinically significative picture in the retina, as alerted by Romano et al. [33]. Last, the most vulnerable to SARS-CoV-2 infection and complications are people aged 65 years and older [34]. The greater prevalence of age-related macular degeneration in this age group [35] can represent a further risk factor for the development of toxic maculopathy.

Although short-term toxicity by ritonavir is far less likely than a long-term impairment, we speculate that in the future we might see some retinopathies in which the role of ritonavir toxicity cannot be ruled out. In similar cases, it would be difficult to discriminate between ritonavir toxic sequelae, possible direct SARS-CoV-2 damage due to its particular ocular tropism [36], and consequences of coadministered hydroxychloroquine.

\section{Conclusion}

This systematic review provides a comprehensive summary of the retinal toxic changes secondary to ritonavir treatment. It might be useful to raise the awareness of patients treated by ritonavir for SARS-CoV-2 infection about the possibility of sight-threatening side effects related to the received therapy so that they can refer to a retina specialist for the research of early signs of retinotoxicity in line with the aims of personalized medicine. 


\section{Appendix}

The case report titled "Bull's eye maculopathy in an HIVpositive patient receiving ritonavir" by Non et al. [37] was excluded for the lack of sufficient clinical details and imaging.

The authors report a case of bull's eye maculopathy pattern in an HIV-positive patient, being treated with ritonavir for 13 years. They describe temporal optic nerve head atrophy in the left eye and areas of macular retinal pigment epithelium (RPE) atrophy with RPE mottling in both eyes. However, they do not provide any fundus autofluorescence image, to confirm the presence of macular atrophy. On the OCT scan, the choroidal hypertransmission is not assessable and the image quality is not high enough to determine the RPE and outer retina status. The diagnosis a previous arterial vascular accident of the retina (temporal nerve head pallor along with central retinal thinning) in the left eye cannot be clearly ruled out on the basis of the information supplied by the authors.

\section{Data Availability}

The data and findings supporting this systematic review are from previous case reports, which have been cited. The processed data are available from the corresponding author upon request.

\section{Conflicts of Interest}

The authors declare that there are no conflicts of interest regarding the publication of this paper.

\section{Authors' Contributions}

Grazia Maria Cozzupoli and Maria Cristina Savastano contributed equally to this work.

\section{References}

[1] Y.-H. Jin, L. Cai, Z.-S. Cheng et al., "A rapid advice guideline for the diagnosis and treatment of 2019 novel coronavirus (2019-nCoV) infected pneumonia (standard version)," Military Medical Research, vol. 7, no. 1, p. 4, 2020.

[2] F. Verdugo-Paiva, A. Izcovich, M. Ragusa, and G. Rada, "Lopinavir-ritonavir for COVID-19: a living systematic review. lopinavir-ritonavir para COVID-19: una revisión sistemática viva," Medwave, vol. 20, no. 6, e7966 pages, 2020.

[3] B. Cao, Y. Wang, D. Wen et al., "A trial of lopinavir-ritonavir in adults hospitalized with severe covid-19," The New England Journal of Medicine, vol. 1-13, 2020.

[4] J. Stebbing, A. Phelan, I. Griffin et al., "COVID-19: combining antiviral and anti-inflammatory treatments," The Lancet Infectious Diseases, vol. 20, no. 4, pp. 400-402, 2020.

[5] C. Flexner, "Dual protease inhibitor therapy in HIV-infected patients: pharmacologic rationale and clinical benefits," $A n$ nual Review of Pharmacology and Toxicology, vol. 40, no. 1, pp. 649-674, 2000.

[6] G. N. Kumar, A. D. Rodrigues, A. M. Buko, and J. F. Denissen, "Cytochrome P450-mediated metabolism of the HIV-1 protease inhibitor ritonavir (ABT-538) in human liver microsomes," The Journal of Pharmacology and Experimental Therapeutics, vol. 277, no. 1, pp. 423-431, 1996.

[7] European Medicines Agency, Annex I: Summary of Product Characteristics, European Medicines Agency, Amsterdam, Netherlands, 2018.

[8] R. K. Vadlapatla, A. D. Vadlapudi, D. Pal, M. Mukherji, and A. K. Mitra, "Ritonavir inhibits HIF- $1 \alpha$-mediated VEGF expression in retinal pigment epithelial cells in vitro," Eye, vol. 28 , no. 1, pp. 93-101, 2014.

[9] T. Kurihara, P. D. Westenskow, S. Bravo, E. Aguilar, and M. Friedlander, "Targeted deletion of Vegfa in adult mice induces vision loss," Journal of Clinical Investigation, vol. 122, no. 11, pp. 4213-4217, 2012.

[10] A.-K. Olsson, A. Dimberg, J. Kreuger, and L. Claesson-Welsh, "VEGF receptor signalling? In control of vascular function," Nature Reviews Molecular Cell Biology, vol. 7, no. 5, pp. 359-371, 2006.

[11] D. Moher, L. Shamseer, M. Clarke et al., "Preferred reporting items for systematic review and meta-analysis protocols (PRISMA-P) 2015 statement," Systematic Reviews, vol. 4, no. 1, 2015.

[12] S. R. Sadda, R. Guymer, F. G. Holz et al., "Consensus definition for atrophy associated with age-related macular degeneration on OCT," Ophthalmology, vol. 125, no. 4, pp. 537-548, 2018.

[13] S. Moola, Z. Munn, C. Tufanaru et al., Chapter 7: Systematic Reviews of Etiology and Risk. In: Joanna Briggs Institute Reviewer's Manual, Joanna Briggs Institute, Adelaide, Australia.

[14] R. H. Roe, J. M. Jumper, V. Gualino et al., "Retinal pigment epitheliopathy, macular telangiectasis, and intraretinal crystal deposits in HIV-positive patients receiving ritonavir," Retina, vol. 31, no. 3, pp. 559-565, 2011.

[15] R. Pinto, M. Vila-Franca, C. Oliveira Afonso, C. Ornelas, and L. Santos, "Ritonavir and bull's eye maculopathy: case report," GMS Ophthalmol Cases, vol. 3, pp. 1-4, 2013.

[16] A. L. Biancardi and A. L. L. Curi, "Retinal toxicity related to long-term use of ritonavir," Retina, vol. 36, no. 1, pp. 229-231, 2016.

[17] E. Papavasileiou, S. Younis, V. Zygoura, C. Quijano, and T. L. Jackson, "RITONAVIR-ASSOCIATED toxicity mimicking retinitis pigmentosa in an HIV-infected patient on highly active antiretroviral therapy," Retinal Cases \& Brief Reports, vol. 11, no. 4, pp. 306-309, 2017.

[18] Y. Tu, R. J. Poblete, B. D. Freilich, M. A. Zarbin, and N. Bhagat, "Retinal toxicity with ritonavir," International Journal of Ophthalmology, vol. 9, no. 4, pp. 640-642, 2016.

[19] C. Faure, M. Paques, and I. Audo, "Electrophysiological features and multimodal imaging in ritonavir-related maculopathy," Documenta Ophthalmologica, vol. 135, no. 3, pp. 241-248, 2017.

[20] L. R. C. Mesquita, M. L. G. Fonseca, R. M. Silva, and E. H. Morizot, "Panretinal ritonavir-induced retinopathy: a report of long-term use," Retinal Cases and Brief Reports, pp. 1-3, 2018.

[21] A. K. Louie and H. N. Jones, "Case report," Optometry and Vision Science, vol. 96, no. 5, pp. 376-381, 2019.

[22] K. A. A. Douglas, V. P. Douglas, and M. M. Moschos, "Ocular manifestations of COVID-19 (SARS-CoV-2): a critical review of current literature," In Vivo, vol. 34, no. 3, pp. 1619-1628, 2020.

[23] Medicines Agency European, "Norvir: EPAR - scientific discussion," 2015, http://www.ema.europa.eu/ docs/en_GB/ document_library/EPAR_Scientific_Discussion/human/0001 27/WC500028725.pdf. 
[24] A. Rismanbaf and S. Zarei, "Liver and kidney injuries in COVID-19 and their effects on drug therapy; a letter to editor," Archives of Academic Emergency Medicine, vol. 8, no. 1, p. e17, 2020.

[25] C. Zhang, L. Shi, and F. Wang, "Liver injury in COVID-19: management and challenges," Lancet, vol. 10, no. 20, pp. 2019-2021, 2020.

[26] Y. Cheng, R. Luo, K. Wang et al., "Kidney disease is associated with in-hospital death of patients with COVID-19," Kidney International, In press, 2020.

[27] P. Colson, J.-M. Rolain, J.-C. Lagier, P. Brouqui, and D. Raoult, "Chloroquine and hydroxychloroquine as available weapons to fight COVID-19," International Journal of Antimicrobial Agents, In press, 2020.

[28] E. Schrezenmeier and T. Dörner, "Mechanisms of action of hydroxychloroquine and chloroquine: implications for rheumatology," Nature Reviews Rheumatology, vol. 16, no. 3, pp. 155-166, 2020.

[29] B. H. Pham and M. F. Marmor, "Sequential changes in hydroxychloroquine retinopathy up to 20 years after stopping the drug," Retina, vol. 39, no. 3, pp. 492-501, 2019.

[30] J. Lochhead, A. Movaffaghy, B. Falsini et al., "The effect of quinine on the electroretinograms of children with pediatric cerebral malaria," The Journal of Infectious Diseases, vol. 187, no. 8, pp. 1342-1345, 2003.

[31] I. Pasaoglu and F. Onmez, "Macular toxicity after short-term hydroxychloroquine therapy," Indian Journal of Ophthalmology, vol. 67, no. 2, pp. 289-292, 2019.

[32] B. N. Hobbs and K. M. Osmotherly, "Hydroxychloroquine: possible covid drug can be toxic to retinas," Medscape, vol. 3-5, 2020.

[33] M. R. Romano, R. Raimondi, A. Montericcio, and D. Allegrini, "Hydroxychloroquine and ritonavir for COVID19 infection: a possible synergic toxicity for retinal pigmented epithelium," Graefe's archive for clinical and experimental ophthalmology = Albrecht von Graefes Archiv Fur klinische und experimentelle Ophthalmologie, p. 4727, 2020.

[34] W. Gardner, D. States, and N. Bagley, "The coronavirus and the risks to the elderly in long-term care," Journal of Aging \& Social Policy, vol. 32, no. 4-5, pp. 310-315, 2020.

[35] J. M. Colijn, G. H. S. Buitendijk, E. Prokofyeva et al., "Prevalence of age-related macular degeneration in Europe: the past and the future," Ophthalmology, vol. 124, no. 12, pp. 1753-1763, 2017.

[36] C. -W. Lu, X. F. Liu, and Z. F. Jia, "2019-nCoV transmission through the ocular surface must not be ignored," Lancet, vol. 395, no. 10224, p. e39, 2020.

[37] L. Non, A. Jeroudi, B. T. Smith, and S. Parsaei, "Bull's eye maculopathy in an HIV-positive patient receiving ritonavir," Antiviral Therapy, vol. 21, no. 4, pp. 365-367, 2016. 